\title{
Distinct mechanisms of form-from-motion perception in human extrastriate cortex
}

\author{
O. Blanke ${ }^{\mathrm{a}, \mathrm{b}, *}$, A. Brooks ${ }^{\mathrm{a}, \mathrm{c}}$, M. Mercier ${ }^{\mathrm{a}}$, L. Spinelli ${ }^{\mathrm{b}}$, \\ M. Adriani ${ }^{a}$, L. Lavanchy ${ }^{b}$, A.B. Safran ${ }^{d}$, T. Landis ${ }^{b}$ \\ ${ }^{a}$ Laboratory of Cognitive Neuroscience, Brain Mind Institute, Ecole Polytechnique Fédérale de Lausanne (EPFL), Lausanne, Switzerland \\ ${ }^{\mathrm{b}}$ Department of Neurology, University Hospital, Geneva, Switzerland \\ ${ }^{\mathrm{c}}$ Laboratory of Perceptual Processing, Southern Cross University, Coffs Harbour, Australia \\ ${ }^{\mathrm{d}}$ Department of Ophthalmology, University Hospital, Geneva, Switzerland
}

Received 22 December 2005; received in revised form 13 July 2006; accepted 23 July 2006

Available online 16 October 2006

\begin{abstract}
The exquisite sensitivity of the human visual system to form-from-motion (FfM) cues is well documented. However, identifying the neural correlates of this sensitivity has proven difficult, particularly determining the respective contributions of different motion areas in extrastriate visual cortex. Here we measured visual FfM perception and more elementary visual motion (VM) perception in a group of 32 patients suffering from acute posterior brain damage, and performed MRI-based lesion analysis. Our results suggest that severe FfM perception deficits without an associated deficit of VM perception are due to damage to ventral occipito-temporal cortex (VOT), whereas associated deficits of FfM and VM perception are due to damage either in proximity to area MT+/V5 or an area including lateral occipital complex (LOC) and VOT. These data suggest the existence of at least three functionally and anatomically distinct regions in human visual cortex that process FfM signals.
\end{abstract}

(C) 2006 Elsevier Ltd. All rights reserved.

Keywords: Visual; Motion; Perception; Neuropsychology; Brain damage

\section{Introduction}

The visual system forms representations of many properties of the environment including information about the shapes or forms of objects and their motion. Form and motion are features with complementary properties and inferring object form involves detecting spatial relationships among features that are generally assumed to be fixed relative to one another. Yet, despite the seemingly complementary nature of form and motion, the two features are closely linked. Motion, for example is an important cue for identifying object boundaries (Jules, 1971). Indeed, the ability to extract the form of objects defined entirely by visual motion cues (hereafter referred to as formfrom-motion or FfM processing) is perhaps one of the more

\footnotetext{
* Corresponding author at: Laboratory of Cognitive Neuroscience, Ecole Polytechnique Fédérale de Lausanne (EPFL), Swiss Federal Institute of Technology, Station 15, 1015 Lausanne, Switzerland. Tel.: +41 21 6939621; fax: +41 216939625

E-mail address: olaf.blanke@epfl.ch (O. Blanke).
}

remarkable achievements of the human visual system. Indeed, there is evidence to indicate the extreme sensitivity of the system to objects defined in this way. Motion contrast serves as an effective cue for the extraction of two- and three-dimensional objects (i.e. Sekuler, 1990; Wist, Ehrenstein, \& Schrauf, 1998).

Studies in brain damaged patients have revealed a functional double dissociation between FfM perception and more elementary visual motion perception (such as direction of motion perception, henceforth referred to as VM processing). These studies described patients with circumscribed brain damage that suffered from deficient VM processing, but normal FfM perception (McLeod, Dittrich, Driver, Perrett, \& Zihl, 1996; Tootell \& Taylor, 1995) or from deficient FfM perception, but normal VM perception (Cowey \& Vaina, 2000; Schenk \& Zihl, 1997a). This double dissociation has been confirmed by Vaina (1989) in a group study. Regan, Giaschi, Sharpe, and Hong (1992) further dissociated between FfM recognition and detection (and normal VM perception) by describing three patients with deficient FfM recognition, but preserved FfM detection (type I loss) and four 
patients with a deficit in FfM recognition and FfM detection (type II loss).

Whilst lesion analyses in patients with deficits in VM perception suggest a circumscribed locus, this is less well described in patients with deficient FfM perception. Deficient VM perception has been linked to bilateral or unilateral damage in lateral temporo-occipital cortex (Barton, Sharpe, \& Raymond, 1995; Blanke, Landis, Mermoud, Spinelli, \& Safran, 2003; Greenlee, Lang, Mergner, \& Seeger, 1995; Vaina, Cowey, Eskew, LeMay, \& Kemper, 2001) in close proximity to area MT+/V5 as defined anatomically (Dumoulin et al., 2000) and functionally by neuroimaging studies (de Jong, Shipp, Skidmore, Frackowiak, \& Zeki, 1994; Dupont et al., 1997; Goebel, Khorram-Sefat, Muckli, Hacker, \& Singer, 1998; Tootell \& Taylor, 1995; Watson et al., 1993) and intracranial electrical stimulation (Blanke, Landis, Safran, \& Seeck, 2002). Deficient FfM perception (with preserved VM perception) has been linked to different brain regions including unilateral temporo-occipital (Vaina, 1989) or temporo-parieto-occipital cortex (Regan et al., 1992), and bilateral parietal (Schenk \& Zihl, 1997a) or temporo-occipital cortex (Cowey \& Vaina, 2000). Lesions in the two group studies led by Vaina (1989) and Regan et al. (1992) were solely defined by computer tomography (CT). In addition, the results of the two studies are difficult to compare since most of the patients in Regan et al.'s (1992) study suffered from chronic and/or neoplastic lesions, while those in the study of Vaina (1989) and Cowey and Vaina (2000) suffered from acute vascular lesions.

Here in a group of 32 patients with acute vascular unilateral posterior brain damage we tested (1) whether we could reconfirm the FfM perception deficits (type I and II loss) and corroborate the evidence (2) that patients with bilateral (Blanke, Vaclavik, Landis, \& Safran, 2003; Vaina, Lemay, Bienfang, Choi, \& Nakayama, 1990; Zihl, von Cramon, \& Mai, 1983) and unilateral brain damage (Vaina \& Gross, 2004) may suffer from a combined FfM and VM deficit. This behavioural analysis was combined with (3) MRI-based lesion analysis in order to examine whether different types of FfM deficits could be attributed to damage of distinct brain areas.

\section{Methods and materials}

\subsection{Patients and control subjects}

Thirty-two acute stroke patients with circumscribed unilateral posterior brain lesions were admitted to the study from a well-defined recruitment area. Eleven healthy subjects formed the control group. Only patients suffering from acute strokes were included in the present study in order to avoid as much as possible functional reorganization related to plasticity changes in patients with chronic lesions. Informed consent was obtained from all patients and the study was conducted in conformity with the Declaration of Helsinki. The visual fields of both eyes were tested for all patients with an OCTOPUS 2000R automated perimeter (Interzeag AG, Switzerland). All patients with visual field defects larger than quadrantanopia were excluded from the study. Visual acuity was normal or corrected to normal in all patients and healthy subjects and no deficits in smooth pursuit eye movements were detected (mild nystagmus was found in two patients). A detailed neuropsychological examination was carried out testing language, praxis, gnosis, executive functions, attention, and memory. Patients presenting cognitive deficits that were likely to bias the comprehension and the execution of our experimental visual tasks were excluded from the study. Patients with language disorders, but spared comprehension and patients with signs of visuo-spatial neglect (appreciated clinically and tested by bell cancellation task and line bisection task) that were clinically judged as mild or moderate were admitted to the study (24 patients had no neglect, the remaining mild or moderate neglect). Orientation discrimination for a stationary letter $\mathrm{E}$ was tested by presenting a hand-held letter $\mathrm{E}$ to the patient in the four different orientations (size and distance the same as used in experimental computerized motion testing). All patients performed this test correctly. None of the patients complained of symptoms related to disturbed motion perception as described in a severely motion blind patient (Zihl et al., 1983). Handedness was evaluated using the Oldfield-Edinburgh questionnaire. All patients carried out the VM task and the FfM task (see below) and their performance was compared to that of a control group of 11 healthy subjects without any neurological impairments. Two patients were left-handed; all other patients and healthy subjects were right-handed. Right hemispheric brain damage was found in 18 patients and left hemispheric brain damage in 14 patients (see Table 1). Three patients had bilateral brain damage that predominated on the right $(n=2)$ or left side $(n=1)$. The 11 control subjects were approximately matched for age, sex and handedness. The mean age of the patients was $62.7 \pm 16.3$ years and of the control group $62.2 \pm 8.9$ years. FfM and VM perception were tested in all patient groups approximately 1 month after lesion onset (range 0.2-3.2 months after lesion onset). For further demographic and clinical details please refer to Table 1 .

\subsection{Stimuli}

\subsubsection{VM task}

Coherent motion stimuli (random dot cinematograms, RDC) were presented on a 20 -in. computer monitor (Sony; frame rate, $70 \mathrm{~Hz}$; 640 pixels $\times 480$ pixels) in black and white in a normally lit room as described previously (Blanke, Landis et al., 2003; Losey, Safran, Mermoud, Michel, \& Landis, 1998). Viewing distance was $100 \mathrm{~cm}$. The stimuli were presented in a borderless square of $12^{\circ} \times 12^{\circ}$ in the central visual field. Each random dot field contained 1000 dots (diameter: $0.68^{\circ}$ ). A percentage of the dots was programmed to be displaced with a velocity of $2 \%$ in the tested direction (signal dots) - comparable to velocities used in previous studies in groups of brain damaged patients (Schenk \& Zihl, 1997b: 1\%; Barton et al., 1995: 3.5\% $/ \mathrm{s}$; Vaina et al., 2001:2.9\% $/ \mathrm{s}$ ). The percentage of coherent motion $(\% \mathrm{CM})$ was defined as the number of signal dots divided by the total number of dots and multiplied by 100 . The remaining dots were noise dots and were plotted at random locations for a random duration (between 67 and $800 \mathrm{~ms}$ ) giving the impression of flickering dots. Dots moving out of the stimulus area reappeared on the opposite side such that density was held constant. The direction of each RDC stimulus in each block was varied randomly between the four cardinal directions (right-left-up-down). An automated staircase algorithm varied the $\% \mathrm{CM}$ in the RDC, starting at $100 \% \mathrm{CM}$ (all dots moving in one direction) (Fig. 1a and b).

\subsubsection{FfM task}

The FfM task was the same (as the VM task) with respect to computer monitor, viewing distance, field of presentation, total number of dots, and dot velocity. A borderless, static form (a capital letter E) was plotted in the center of the $12^{\circ} \times 12^{\circ}$ random dot field. The size of the letter was $6^{\circ} \times 6^{\circ}$ and was defined by 250 signal dots. The remaining 750 dots were plotted at random locations in the area outside the letter (for a random duration) giving the impression of flickering dots. Thus, total number of dots was the same (1000 dots) in the VM and the FfM task. Within the central area of $6^{\circ} \times 6^{\circ}$ the letter was plotted in different proportions of signal and noise dots: the ratio of signal dots to noise dots was manipulated for the 250 dots in the central area (of $6^{\circ} \times 6^{\circ}$ ) while the 750 dots outside the central area (of $6^{\circ} \times 6^{\circ}$ ) were always noise dots. Thus, the percentage of coherent motion $(\% \mathrm{CM})$ was defined as the number of signal dots divided by the total number of 250 dots (inside the central area of $6^{\circ} \times 6^{\circ}$ ) and multiplied by 100 . The remaining dots inside the central area of $6^{\circ} \times 6^{\circ}$ were noise dots and were plotted at random locations (inside the central area of $6^{\circ} \times 6^{\circ}$ ) for a random duration (between 67 and $800 \mathrm{~ms}$ ) giving the impression of flickering dots as all dots outside the central area. Overall stimulus density was equivalent with the VM task, thus ensuring task comparability. As in the VM task, the direction of each RDC stimulus in each block was varied randomly between the four cardinal directions of motion and four orientations (right-left-up-down). An automated staircase algorithm varied the \% CM in the RDC, starting at $100 \% \mathrm{CM}$ (all dots moving in one direction) (Fig. 1c-f). 
Table 1

Patient demographic and clinical data

\begin{tabular}{|c|c|c|c|c|c|c|c|c|}
\hline \multirow[t]{2}{*}{ Patients } & \multirow[t]{2}{*}{ Age } & \multirow[t]{2}{*}{ Gender } & \multirow[t]{2}{*}{ Handedness } & \multicolumn{4}{|l|}{ Lesion } & \\
\hline & & & & Duration (month) & Side & Location & Visual field & \\
\hline A-1 & 59 & $\mathrm{~F}$ & $\mathrm{~L}$ & 0.3 & $\mathrm{~L}$ & $\mathrm{P}-\mathrm{O}$ & FULL & Type I loss \\
\hline A-2 & 57 & $\mathrm{~F}$ & $\mathrm{R}$ & 0.3 & $\mathrm{R}$ & $\mathrm{P}-\mathrm{O}$ & FULL & Type I loss \\
\hline A- 8 & 49 & M & $\mathrm{R}$ & 0.3 & $\mathrm{R}$ & O-P-T & FULL & Type I loss \\
\hline A-3 & 74 & M & $\mathrm{R}$ & 0.5 & $\mathrm{R}$ & $\mathrm{T}-\mathrm{O}$ & LSQ & Type II loss \\
\hline A-4 & 79 & M & $\mathrm{R}$ & 2.9 & $\mathrm{~L}$ & $\mathrm{~T}-\mathrm{O}$ & RIQ & Type II loss \\
\hline A-5 & 46 & $\mathrm{~F}$ & $\mathrm{R}$ & 0.4 & $\mathrm{R}$ & $\mathrm{T}-\mathrm{O}$ & RSQ & Type II loss \\
\hline A-6 & 90 & M & $\mathrm{R}$ & 0.6 & $\mathrm{R}$ & $\mathrm{T}-\mathrm{O}$ & FULL & Type II loss \\
\hline A-7 & 78 & $\mathrm{~F}$ & $\mathrm{~L}$ & 0.9 & $\mathrm{~L}$ & O-P-T & FULL & Type II loss \\
\hline B-13 & 59 & M & $\mathrm{R}$ & 1.2 & $\mathrm{R}$ & $\mathrm{O}$ & RIQ & Type III loss \\
\hline B-4 & 93 & M & $\mathrm{R}$ & 0.8 & $\mathrm{~B}(\mathrm{R})$ & $\mathrm{P}$ & FULL & Type III loss \\
\hline B-12 & 50 & M & $\mathrm{R}$ & 0.4 & $\mathrm{R}$ & P-O & LIQ & Type III loss \\
\hline B-3 & 80 & M & $\mathrm{R}$ & 1.2 & $\mathrm{R}$ & $\mathrm{P}-\mathrm{O}(\mathrm{O})$ & LIQ & Type III loss \\
\hline B-1 & 60 & M & $\mathrm{R}$ & 1.2 & $\mathrm{R}$ & P-O (O) & LIQ & Type III loss \\
\hline B-8 & 72 & $\mathrm{~F}$ & $\mathrm{R}$ & 0.5 & B (L) & O-P-T & RIQ & Type III loss \\
\hline B-7 & 73 & $\mathrm{~F}$ & $\mathrm{R}$ & 0.4 & $\mathrm{~L}$ & $\mathrm{~T}-\mathrm{O}$ & RIQ & Type III loss \\
\hline B-9 & 49 & $\mathrm{~F}$ & $\mathrm{R}$ & 4.1 & $\mathrm{~L}$ & $\mathrm{~T}-\mathrm{O}$ & FULL & Type III loss \\
\hline B-15 & 75 & $\mathrm{~F}$ & $\mathrm{R}$ & 0.4 & $\mathrm{~L}$ & $\mathrm{~T}-\mathrm{O}$ & RSQ & Type III loss \\
\hline B-10 & 63 & $\mathrm{~F}$ & $\mathrm{R}$ & 0.7 & $\mathrm{~L}$ & $\mathrm{~T}-\mathrm{O}$ & FULL & Type III loss \\
\hline B-5 & 31 & $\mathrm{~F}$ & $\mathrm{R}$ & 2 & $\mathrm{R}$ & $\mathrm{T}-\mathrm{O}$ & LSQ & Type III loss \\
\hline B-2 & 77 & $\mathrm{~F}$ & $\mathrm{R}$ & 0.7 & $\mathrm{R}$ & P-P & FULL & Type IV loss \\
\hline B-6 & 72 & M & $\mathrm{R}$ & 3.2 & $\mathrm{R}$ & $\mathrm{P}-\mathrm{O}-\mathrm{T}$ & LIQ & Type IV loss \\
\hline B-11 & 40 & M & $\mathrm{R}$ & 2 & $\mathrm{~B}(\mathrm{R})$ & $\mathrm{P}-\mathrm{O}$ & LIQ & Type IV loss \\
\hline B-14 & 67 & M & $\mathrm{R}$ & 1 & $\mathrm{R}$ & $\mathrm{O}$ & LSQ & Type IV loss \\
\hline B-16 & 65 & $\mathrm{~F}$ & $\mathrm{R}$ & 0.3 & $\mathrm{R}$ & $\mathrm{O}$ & L-SCOTOMA & Type IV loss \\
\hline $\mathrm{C}-1$ & 75 & $\mathrm{~F}$ & $\mathrm{R}$ & 1 & $\mathrm{~L}$ & $\mathrm{~T}-\mathrm{O}$ & RSQ & Group V \\
\hline $\mathrm{C}-2$ & 75 & M & $\mathrm{R}$ & 0.2 & $\mathrm{R}$ & $\mathrm{T}-\mathrm{O}$ & LSQ & Group V \\
\hline $\mathrm{C}-3$ & 72 & M & $\mathrm{R}$ & 0.2 & $\mathrm{~L}$ & $\mathrm{P}-\mathrm{O}$ & R-SCOTOMA & Group V \\
\hline C-4 & 27 & $\mathrm{~F}$ & $\mathrm{R}$ & 2.7 & $\mathrm{R}$ & $\mathrm{T}-\mathrm{O}$ & FULL & Group V \\
\hline $\mathrm{C}-5$ & 41 & M & $\mathrm{R}$ & 0.8 & $\mathrm{~L}$ & O-P-T & RSQ & Group V \\
\hline C-6 & 58 & $\mathrm{~F}$ & $\mathrm{R}$ & 1.3 & $\mathrm{~L}$ & $\mathrm{P}-\mathrm{O}$ & FULL & Group V \\
\hline $\mathrm{C}-7$ & 42 & M & $\mathrm{R}$ & 0.2 & $\mathrm{~L}$ & $\mathrm{O}-\mathrm{T}$ & FULL & Group V \\
\hline C-8 & 57 & M & $\mathrm{R}$ & 1.6 & $\mathrm{~L}$ & O-P-T & FULL & Group V \\
\hline
\end{tabular}

M, male; F, female; r, right-handed; R, right hemisphere lesion; L, left hemisphere lesion; P, parietal; O, occipital; T, temporal; LIQ, left inferior quadrantanopia; RIQ, right inferior quadrantanopia.

\subsection{Procedure}

In the VM task, subjects were asked to indicate verbally whether they perceived motion direction as right, left, up, down. In the FfM task, subjects were asked to indicate verbally whether they perceived the three horizontal bars of the letter $\mathrm{E}$ to be pointing to the right, left, up, down. However, patients frequently and repeatedly replied that they did not perceive any directed motion and could not indicate any specific direction. We thus modified the paradigm to the patient's comfort and carried out a five-alternative forced-choice paradigm in which the patients had to indicate verbally either the perceived direction or that they did not perceive the direction of motion. This combination of motion direction discrimination and detection was carried out in patients and normal subjects (Blanke, Landis et al., 2003). For each trial, if the answer of the subject was correct the $\% \mathrm{CM}$ was decreased. If it was incorrect, \% $\mathrm{CM}$ was increased (either for incorrect direction or "no" response). Four independent staircases (one for each direction of motion) were randomly interleaved for both tasks. The four staircases were continued until five response reversals had occurred for each tested direction. The staircase steps had a scaling factor of 0.67 . Thus, the $\% \mathrm{CM}$ values (staircase steps) were generated using steps that decreased by $1 / 3$ of the previous higher value $(100 \times 2 / 3=66.7 ; 66.7 \times 2 / 3=44.4 ; 44.4 \times 2 / 3=29.6 ; 29.6 \times 2 / 3=19.8$, etc.). The procedure ( 5 reversals) estimates about $0.8 \% \mathrm{CM}$ for the VM task and $8.7 \% \mathrm{CM}$ in the FfM task. The mean of the last three reversals was taken as the $\% \mathrm{CM}$ threshold. The mean of these four directional thresholds for each subject provided the threshold for VM and FfM perception. Subjects were instructed to look at the center of the screen and to refrain from making eye movements. Fixation was monitored by the examiner. Patients who could not maintain fixation were excluded from analysis (we cannot exclude that small amplitude eye movements might have occurred during some trials in some of the examined patients). No feedback about correctness of the response was provided. Subjects gave their answer verbally and the examiner recorded the response. The rate of trial presentation was controlled by the examiner and adjusted to patient comfort. Testing took approximately $30 \mathrm{~min}$ and was carried out in one session. With respect to the performance of the control subjects, the patients' performance was evaluated using a $t$-distribution since our sample was quite small $(n<50)$. Upper $99 \%$ prediction intervals were derived. Patients with \% CM thresholds falling above these limits of the mean \% CM threshold were considered pathological threshold elevations.

\subsection{Lesion analysis}

Lesion analysis was carried out as described previously (Blanke, Landis et al., 2003). All brain lesions were delineated by magnetic resonance imaging (MRI). MRI was performed with a 1.5 Tesla Eclipse system (Marconi Medical Systems, Cleveland, OH). Lesions were drawn on the original MRIs and subsequently normalized into Talairach space (Talairach \& Tournoux, 1988). 
(a)

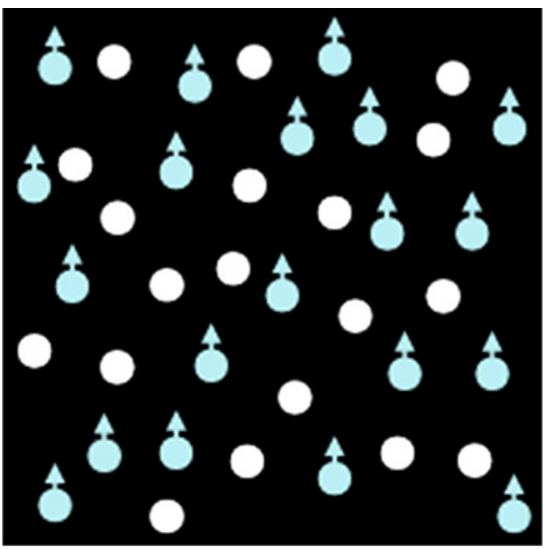

(c)

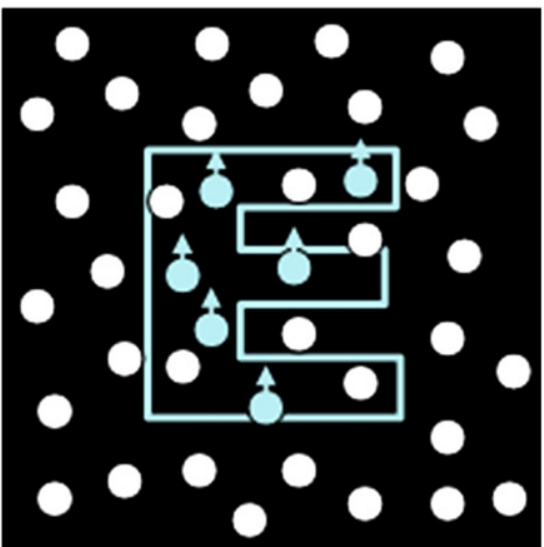

(e)

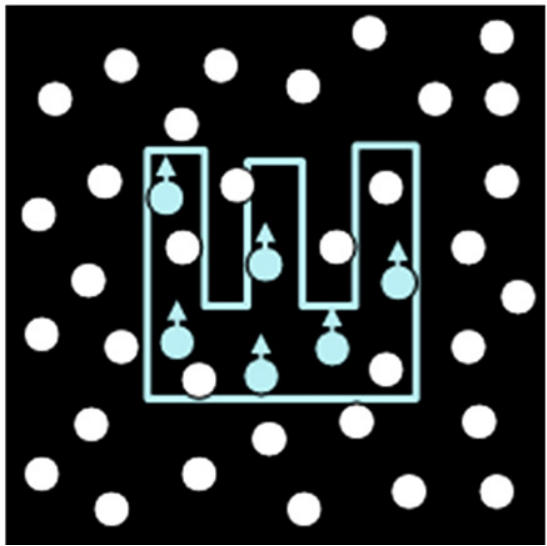

(b)

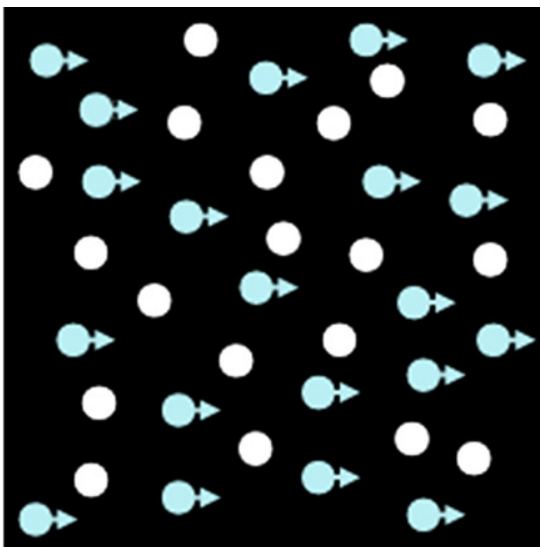

(d)
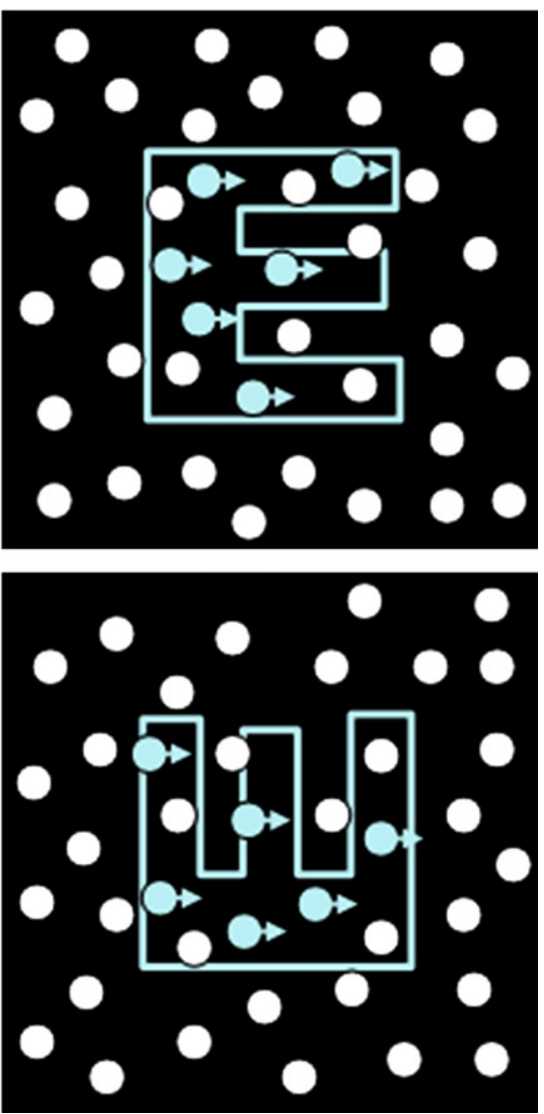

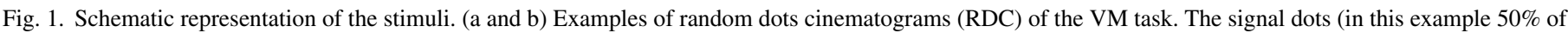

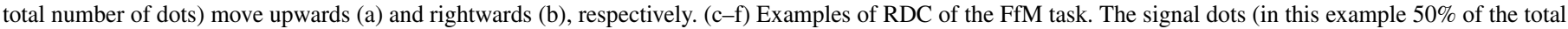

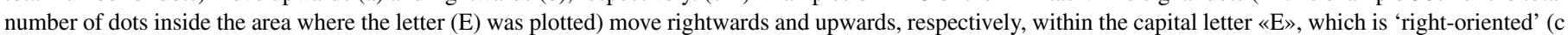
and d) or 'up-oriented' (e and f).

For each patient of a given group (groups $\mathrm{I}-\mathrm{V}$ ), the individual lesion was mapped onto the hemisphere that was most often affected in that group. Thus, in groups I-IV lesions were mapped on the right hemisphere and in group $\mathrm{V}$ on the left hemisphere. In patients with bilateral damage, only the predominantly lesioned hemisphere was included in the lesion analysis (in patients B-4 and B-11 the right hemisphere lesion and in patient B-8 the left hemisphere lesion; Table 1). Three-dimensional rendering and superimposing of the individual lesions was carried out using AVS software (Advanced Visual Systems, MA, USA). Area of greatest lesion overlap was defined with respect to sulcal and gyral anatomy, as well as Talairach coordinates. The Talairach coordinates of the lesion overlap area were calculated for each group as the range of the $x, y$, and $z$ coordinates of a box that included the area of lesion overlap.

\section{Results}

\subsection{Controls}

Normal discrimination thresholds in the FfM task were $8.7 \pm 4.4 \% \mathrm{CM}$ (mean \pm 1 standard deviation) and in the VM task $0.8 \pm 0.4 \%$ CM. $99 \%$ prediction intervals were calculated for both tasks and are indicated in Fig. 2 by dashed lines. Threshold values above this limit were considered pathological as reported elsewhere (Barton et al., 1995; Blanke, Landis et al., 2003; Regan et al., 1992). 


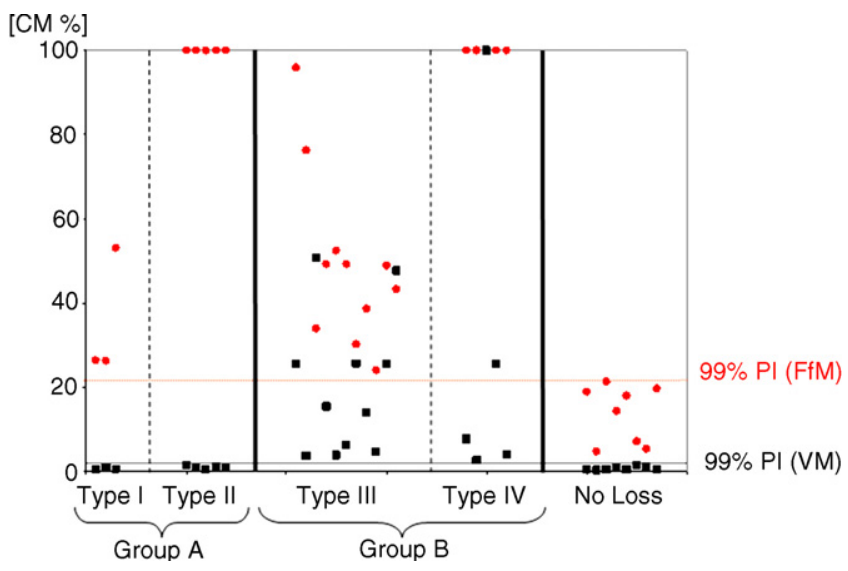

Fig. 2. Psychophysical thresholds for FfM and VM perception. Psychophysical thresholds for FfM perception (grey) and VM perception (black) are given for each patient separately. Patients with type I loss, type II loss (belonging to group A), type III loss, type IV loss (belonging to group B), and no loss (group V) are depicted together.

\subsection{Patients}

Depending on their performance in the FfM and VM task and following the classification by Regan et al. (1992), patients were classified into five groups. The first group consisted of patients who had abnormally elevated psychophysical thresholds in the FfM task, but could discriminate the motion-defined form at least at the highest threshold value $(100 \% \mathrm{CM}$ - FfM discrimination deficit) associated with normal VM perception (type I loss after Regan et al., 1992). The second group consisted of patients who had abnormally elevated psychophysical thresholds in the FfM task, but could not discriminate the motion-defined form at the highest threshold value (severe FfM discrimination deficit) associated with normal VM perception (type II loss after Regan et al., 1992). The third group consisted of Type III loss patients: patients who suffered from a FfM discrimination deficit but in addition from a VM discrimination deficit. The fourth group consisted of Type IV loss patients: patients who suffered from a severe FfM discrimination deficit associated with a VM discrimination deficit. Type I loss was found in 3 patients, type II loss in 5 patients, type III loss in 11 patients, and type IV loss in 5 patients. The fifth group consisted of eight patients with normal FfM and VM discrimination. We found no patient with normal FfM perception and pathological VM perception. Clinical details of each patient group are given in Table 1. Type of FfM and VM deficit and number of patients per group are summarized in Table 2.

Grouping between patients with severe motion discrimination deficits $(100 \% \mathrm{CM}$ threshold not discriminated; groups II and IV) and those with moderate motion discrimination deficits (elevated \%CM thresholds; groups I and III) was based on clinical and phenomenological differences and not statistical differences (because we applied a five alternative forced choice test rather than a four alternative forced choice discrimination test in combination with an additional detection test). Accordingly, we also analyzed VM and FfM performance in two larger patient groups. Group A (8 patients) consisted of the patients (from groups I and II) who presented a normal performance in the VM task, but had abnormally elevated psychophysical thresholds in the FfM task. Group B consisted of the patients (from groups III and IV; 16 patients) who presented abnormally elevated psychophysical thresholds in the VM task and in the FfM task.

\subsubsection{Threshold analysis}

In the patients with type I loss, the FfM thresholds ranged from 26.3 to $53.0 \% \mathrm{CM}$. VM thresholds ranged from 0.6 to $1.0 \% \mathrm{CM}$ (Fig. 2). Mean thresholds ( \pm standard deviation) were $35.3 \pm 15.3 \%$ (FfM) and $0.7 \pm 0.2 \% \mathrm{CM}(\mathrm{VM})$. All patients perceived the letter in the FfM task at the highest threshold value $(100 \% \mathrm{CM})$.

None of the five patients with type II loss were able to perceive the letter in the FfM task at the highest threshold value $(100 \% \mathrm{CM})$, although all easily recognized the letter when it was presented as a stationary object. In these patients no measurements of FfM discrimination were carried out (in Fig. 2 the FfM thresholds are plotted as $100 \% \mathrm{CM}$ ). Yet, all five patients performed the VM task. Moreover, VM perception was normal in all patients with type II loss and ranged from 0.7 to $1.5 \% \mathrm{CM}$ (mean: 1.0; standard deviation: $0.3 \% \mathrm{CM}$ ).

The largest group of patients $(n=11)$ suffered from type III loss, i.e. a FfM discrimination deficit and a VM discrimination deficit (see Fig. 2). FfM thresholds ranged from 24.1 to 95.5\% CM (mean: 49.3\% CM; standard deviation: 20.7\% CM) and $\mathrm{VM}$ thresholds ranged from 3.7 to $50.7 \% \mathrm{CM}$ (mean: 20.3\%CM; standard deviation: $16.7 \% \mathrm{CM}$ ). All patients perceived the letter in the FfM task at the highest threshold value $(100 \% \mathrm{CM})$.

The five patients with a type IV loss suffered from a severe FfM discrimination deficit and a VM discrimination deficit. All

Table 2

Patients' performance classification

\begin{tabular}{lllllll}
\hline & Groups & $\begin{array}{l}\text { Severe FfM } \\
\text { discrimination deficit }\end{array}$ & $\begin{array}{l}\text { FfM discrimination } \\
\text { deficit }\end{array}$ & $\begin{array}{l}\text { Severe VM } \\
\text { discrimination deficit }\end{array}$ & $\begin{array}{l}\text { VM discrimination } \\
\text { deficit }\end{array}$ & $\begin{array}{l}\text { Total number of patients } \\
\text { for each group }\end{array}$ \\
\hline A & Type I loss & - & + & - & - & 3 \\
& Type II loss & + & + & - & - & 5 \\
B & Type III loss & - & + & - & + & 11 \\
& Type IV loss & + & + & - & + & 5 \\
C & Group V & - & - & - & - & 8
\end{tabular}

Patients were classified into five different subtypes according to their performance in the VM task and in the FfM task. (+) The deficit is present; (-) the deficit is absent. 
patients performed the FfM task, but none of the patients with type IV loss were able to discriminate the FfM stimulus at the highest threshold value $(100 \% \mathrm{CM})$. All patients perceived the letter in the FfM task at the highest threshold value (100\%CM) and easily recognized the letter when it was presented as a stationary object (in Fig. 2 the FfM thresholds of these patients are plotted as $100 \% \mathrm{CM}$ ). The $\mathrm{VM}$ thresholds ranged from 2.7 to 100\% CM (mean: 28.0\% CM; standard deviation: 41.3\%CM).

The FfM thresholds in the eight patients from group $\mathrm{V}$ ranged from 5.5 to $21.4 \% \mathrm{CM}$ (mean: $13.7 \% \mathrm{CM}$; standard deviation: $6.9 \% \mathrm{CM}$ ) and $\mathrm{VM}$ thresholds ranged from 0.4 to $1.2 \% \mathrm{CM}$ (mean: $0.7 \% \mathrm{CM}$; standard deviation: $0.4 \% \mathrm{CM}$ ).

The eight patients of group A (combined analysis of the patients from groups I and II) had a mean psychophysical threshold of $0.9 \% \mathrm{CM}( \pm 0.3 \% \mathrm{CM})$ in the $\mathrm{VM}$ task and of $75.7 \% \mathrm{CM}$ $( \pm 34.5 \% \mathrm{CM})$ in the FfM task. The 16 patients of group B (combined analysis of the patients from groups III and IV) had a mean psychophysical threshold of $22.7 \% \mathrm{CM}( \pm 25.6 \% \mathrm{CM})$ in the $\mathrm{VM}$ task and of $65.1 \% \mathrm{CM}( \pm 29.6 \% \mathrm{CM})$ in the FfM task.

\subsubsection{Lesion analysis}

Of the 24 patients with deficient FfM perception (types I-IV) the majority (67\%) suffered from right hemispheric lesions (Table 1). All patient groups included patients with right and left hemispheric lesions except patients with type IV loss. All patients with type IV loss had right hemispheric or bilateral lesions. Bilateral lesions were found in patients with type III $(n=2)$ and type IV loss $(n=1$; Table 1$) .75 \%$ of patients from group V suffered from left hemispheric lesions.

Fig. 3 shows the results of lesion overlap analysis for all patient groups. All lesions were mapped onto the right hemisphere. Lesion overlap in patients with type II loss centered in basal temporo-occipital cortex, in lingual, parahippocampal, posterior fusiform gyri and underlying white matter (range: 0 ,
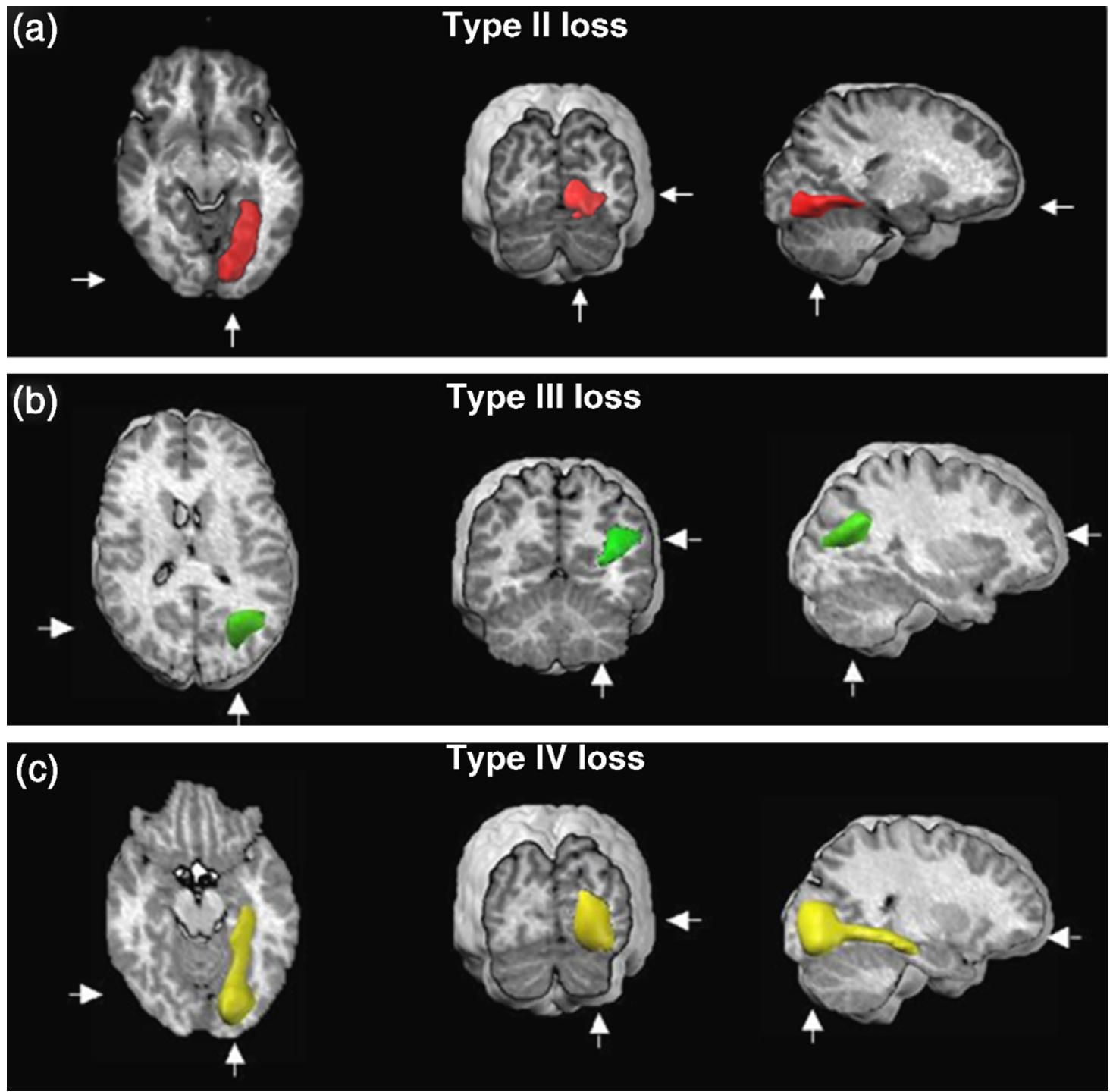

Fig. 3. Lesion analysis in patients with impaired FfM motion perception. Lesion analysis of patients with type II loss (a), with type III loss (b), and with type IV loss (c) is shown. The region of lesion overlap is shown for all groups in transverse (left), coronal (middle), and sagital (right) planes (white arrows indicate the respective position of the chosen planes). The present analysis localized lesion overlap in patients with type II loss at/near VOT, in patients with type III loss at/near area MT/V5, and in patients with type IV loss at/near LOC (see text for further discussion). 

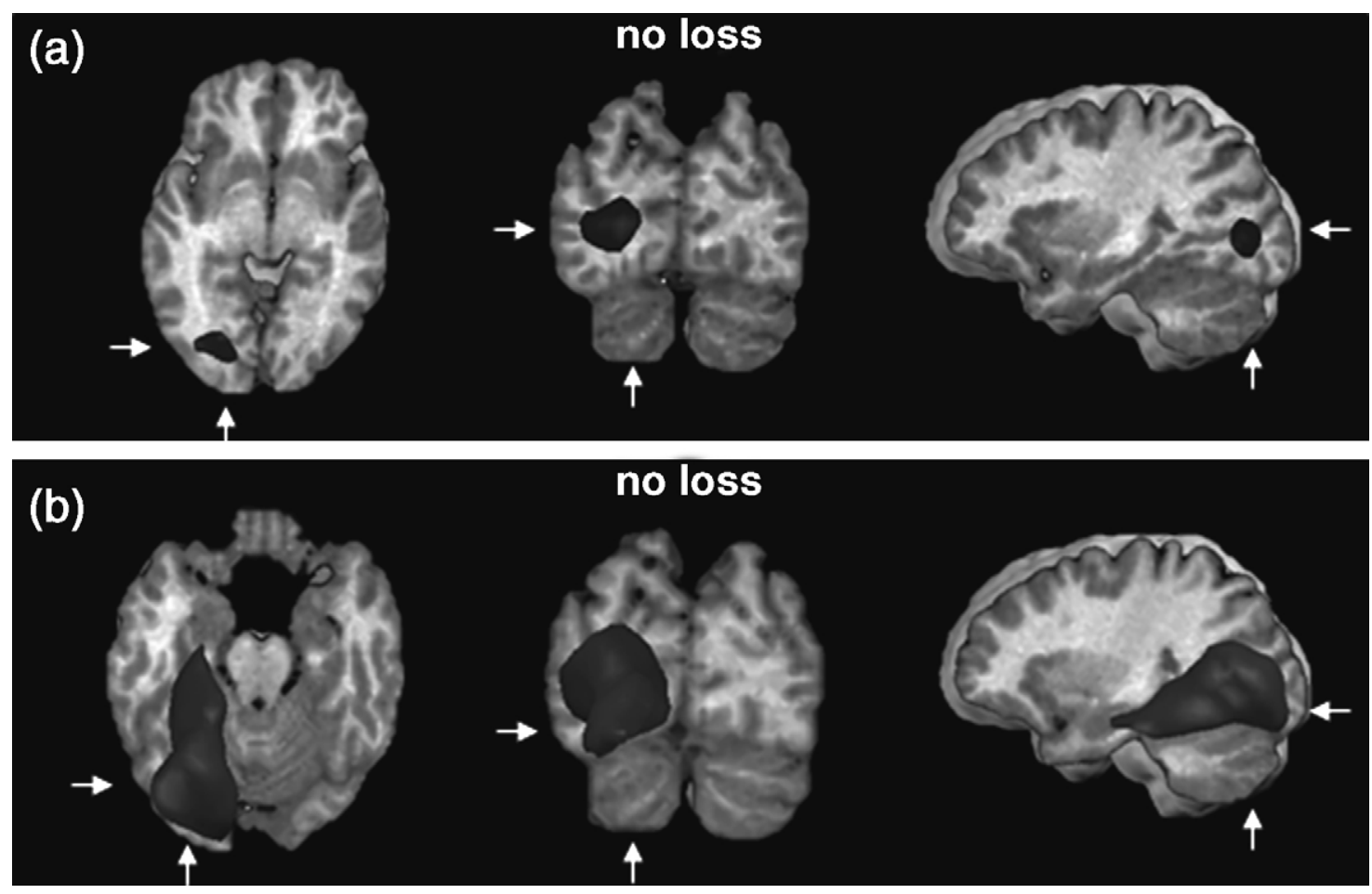

Fig. 4. Lesion analysis in patients with normal FfM motion perception. The lesion overlap (a) and the range (b) of cerebral regions affected in patients with normal motion perception (group V) are shown. The region of lesion overlap and the lesion range are shown in transverse (left), coronal (middle), and sagital (right) planes (white arrows indicate the respective position of the chosen planes).

$31[x] ;-29,-89[y] ;-24,3[z])$. Lesion overlap in patients with type II loss thus centered in ventral occipito-temporal cortex (or VOT; Grill-Spector \& Malach, 2004) including area V4 (Bartels $\&$ Zeki, 2000) and included $60 \%$ of the patients in this group (Fig. 3a). Lesion overlap in patients with type III and IV loss centered in other posterior brain areas. This is shown in Fig. $3 b$ and $\mathrm{c}$.

Lesion overlap in patients with type III loss centered more laterally and dorsally at the junction of parietal, occipital, temporal cortex, and underlying white matter. Lesion overlap included angular, supramarginal, and adjacent occipital gyri (Fig. 3b; range: $24,50[x] ;-45,-70[y] ; 5,29[z])$. Lesion overlap is thus localized in the dorsal stream in proximity to area MT+/V5 (Barton et al., 1995; Cowey \& Vaina, 2000; Dupont, Orban, De
Bruyn, Verbruggen, \& Mortelmans, 1994; Ferber, Humphrey, \& Vilis, 2003; Johansson, 1973; Talairach \& Tournoux, 1988; Wang et al., 1999) and included $45 \%$ of the patients in this group.

Lesion overlap in patients with type IV loss was more widespread and centered in basal and lateral occipito-temporal cortex and was localized between the damage that was observed in patients with type II and III loss (range: $8,39[x] ;-16,-95$ $[y] ;-25,22[z])$. It included fusiform, lingual, inferior and middle occipital gyri, as well as underlying white matter and was in proximity to lateral occipital complex (or LOC) and VOT (Greenlee et al., 1995; Fig. 3c). The overlap area consisted of $60 \%$ of the patients in this group. No area of lesion overlap was found in patients with type I loss.

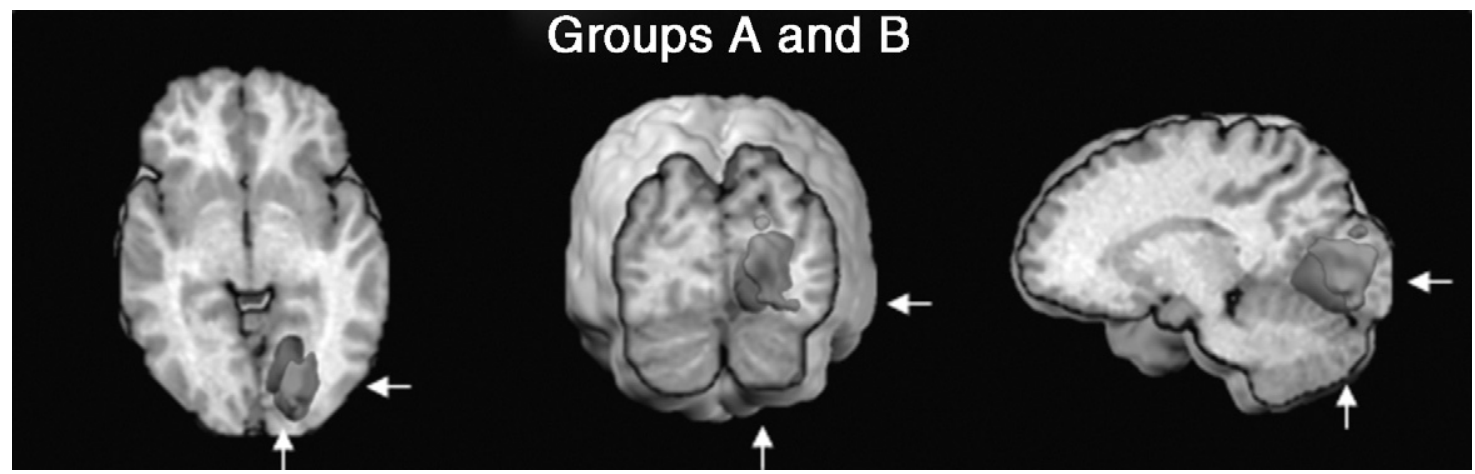

Fig. 5. Lesion overlap analysis of groups A and B. Lesion analysis of patients in group A (normal performance in VM task and deficient performance in FfM task) and patients in group B (deficient performance in VM task and FfM task). The region of lesion overlap is shown for all groups in transverse (left), coronal (middle), and sagital (right) planes (white arrows indicate the respective position of the chosen planes). 
Fig. 4a shows lesion overlap in patients with normal motion perception (group V) and centered in the ventral occipitotemporal cortex that included the anterior cuneus and the lingual gyrus (Fig. 4a; range: $-12,-42[x] ;-66,-86[y] ;-9,14[z])$. The overlap area consisted of $50 \%$ of the patients in this group. We also plotted the range of lesions for all patients from group $\mathrm{V}$ (Fig. 4b). All lesions of group V were mapped onto the left hemisphere.

Fig. 5 shows lesion overlap analysis for groups A and B (all lesions were mapped onto the right hemisphere). Lesion overlap in patients from group A (type I and type II loss) centered in basal temporo-occipital cortex, in lingual, parahippocampal, posterior fusiform gyri and underlying white matter (Fig. 5, black area; range: $0,46[x] ;-26,-88[y] ; 16,-26[z])$, while lesion overlap in patients from group B (type III and type IV loss) centered more laterally in lateral occipito-temporal cortex, extending dorsally and including underlying white matter (Fig. 5, white area; range: $+10,+32[x] ;-47,-93[y] ; 21,-22[z])$.

\section{Discussion}

\subsection{Selective impairment of FfM perception}

Our results in patients with type I and II loss corroborate and extend previous findings about deficient FfM perception in patients with unilateral posterior brain damage (Regan et al., 1992; Vaina, 1989). Although the different authors applied different behavioural FfM paradigms, the present data in patients with type I and II loss suggest that mechanisms of FfM perception are independent of mechanisms of more elementary VM perception: a notion also put forward by Cowey and Vaina (2000) in a study of a patient with bilateral posterior brain damage. Thus, Regan et al. (1992) used 10 different letters for letter recognition and detection using different directions of motion for the letter and the background, whereas Vaina (1989) used six different forms for the form recognition task presenting the form on a stationary background. Here, we used one letter and presented it in four different orientations and directions on a stationary, but flickering, background. In addition, these previous studies used different VM tasks: Regan et al. (1992) measured speed thresholds (right-left discrimination) whereas Vaina (1989) used a same-different speed discrimination task. Given that the FfM and VM paradigms used in the different studies and the present study differed and that different aspects of VM perception such as speed and coherence may be affected differently by brain damage (Vaina, Cowey, Jakab, \& Kikinis, 2005) it might thus be possible that observed dissociations between FfM and VM perception due to brain damage may also vary depending on what stimuli are used.

Previous lesion analysis in group studies was based on CT and linked deficient FfM perception (with normal VM perception) either to unilateral lesions of temporo-occipital (Vaina, 1989) or temporo-parieto-occipital (Regan et al., 1992) cortex, or to bilateral lesions of occipito-temporal cortex (Cowey \& Vaina, 2000). It was thus not clear whether deficient FfM perception is linked to ventral or dorsal stream damage, or whether damage to either stream may lead to deficient FfM perception.
The present study allowed a more detailed lesion analysis and found the area of maximal lesion overlap in patients with a severe FfM deficit (type II loss) in the ventral stream in ventral occipito-temporal cortex (or VOT) including V4. Although it is possible that due to perifocal edema, brain damage extended beyond this area, the present data are concordant with the results of Vaina (1989) and Cowey and Vaina (2000). Visual inspection of the lesion analyses of patients with a FfM recognition deficit from Regan et al. (1992) suggests that VOT was also damaged in many patients from this group. This lesion location in VOT also agrees with neuroimaging studies that have consistently shown that several well-defined areas in VOT are active during the perception and recognition of complex forms such as objects and faces (Grill-Spector \& Malach, 2004). Moreover, VOT has been shown to be activated by FfM stimuli (Gulyas, Heywood, Popplewell, Roland, \& Cowey, 1994; Wang et al., 1999). It can thus be assumed that brain damage to neuronal populations that encode form-from-motion signals in VOT led to the present deficits in FfM perception because static form perception and VM perception were normal in these patients. Alternatively, this deficit (as well as the deficits described below) might also be due to functional disconnection between visual areas involved in FfM perception. Deficits in patients with type II loss cannot be explained by severe language difficulties or hemianopia as patients with such deficits were excluded from the study. Although we cannot exclude that the severe deficit in patients with type II loss was due to a criterion bias instead of a severe threshold elevation (i.e. patients refusing to respond) this is not very likely because patients with type II loss did not refuse to respond in the VM-task (using an identical paradigm except for the stimulus presented). Importantly, this was the case for all patients in group II. Finally, all patients from group II not only performed the VM-task, but also had normal VM perception thresholds. Although, Vaina (1989) suggested that only right occipito-temporal lesions lead to deficient FfM perception, Regan et al. (1992) and the present study observed such deficits for patients with damage to the left or the right posterior hemisphere although right hemisphere damage was more frequent. The absence of impaired FfM perception in Vaina's (1989) left hemispheric group might thus have been due to the fact that fewer patients with left temporo-occipital lesions were investigated, and were therefore analyzed together with patients suffering from left occipito-parietal lesions (Vaina, 1989). Our lesion analysis found no area of lesion overlap in patients with type I loss (FfM discrimination deficit). We argue that this might be due to the small number of patients in this group, to the mild FfM deficits without associated VM deficits (only one patient had a 2-3-fold increase in FfM thresholds whereas the other two patients were just above the limits of normal FfM perception), or to the fact that type II loss might be associated with damage to different brain areas and/or is a rather unspecific motion perception deficit.

To summarize, our data suggest that damage to right or left VOT may lead to a severe deficit in FfM perception that is independent from static form perception and independent from VM perception. 


\subsection{Selective impairment of VM perception}

Interestingly, we found no patient who was deficient in VM perception whilst retaining normal FfM processing capabilities. This dissociation was reported previously by Vaina (1989) in five patients with dorsal stream damage in right parieto-occipital cortex. Although we cannot exclude that methodological differences might account for these differences, ${ }^{1}$ inspection of Fig. 2 reveals that two patients with type III loss showed mild threshold elevations in the FfM task and moderate to severe elevations in the VM task. These patients might thus be compared to the abovementioned patients reported by Vaina (1989). Patient B-7 had a four-fold threshold elevation in the FfM task $(30.3 \% \mathrm{CM})$ and a 30 -fold elevation $(25.6 \% \mathrm{CM})$ in VM task. Similar findings were obtained in patient B-12 (FfM: 34.1\%CM; VM: $50.7 \% \mathrm{CM}$ ). Patient B-7 suffered from unilateral and patient B-12 from bilateral brain damage to dorsal stream areas and mainly to parieto-occipital cortex consistent with the observation by Vaina (1989). This is also consistent with previous single case studies in patients with bilateral lesions to dorsal stream areas (including occipital, temporal, and parietal cortex) (McLeod et al., 1996; Vaina et al., 1990) who exhibited preserved FfM perception despite severely impaired VM perception. Neuroimaging studies have consistently shown that area MT+/V5 and several areas in posterior parietal cortex are strongly activated by VM stimuli (de Jong et al., 1994; Dupont et al., 1997; Goebel et al., 1998; Tootell \& Taylor, 1995; Watson et al., 1993), while these areas were less activated by FfM stimuli (Ferber et al., 2003; Gulyas et al., 1994; Wang et al., 1999).

To summarize, these data suggest that spared or relatively spared FfM perception in patients with severely deficient VM might be related to dorsal stream damage including area MT+/V5 (McLeod et al., 1996; Vaina et al., 1990) as well as adjacent areas in occipito-parietal cortex (Vaina, 1989, present study).

\subsection{Associated impairments of FfM and VM perception}

The association of impaired VM with FfM perception has been described previously in studies reporting single patients with bilateral brain damage (Blanke, Landis et al., 2003; Vaina et al., 1990; Zihl et al., 1983) and in a group of four patients with unilateral brain damage (Vaina \& Gross, 2004). The present results confirm in a larger group of patients that unilateral brain damage may lead to a combined deficit of VM and FfM perception (type III and IV loss) and suggest that two types can be dissociated functionally and anatomically from patients with type II loss.

We linked mild to moderate deficits in FfM perception that were associated with a VM deficit to damage in temporo-parietal cortex in close proximity to MT+/V5 (type III loss), whereas severe deficits in FfM perception that were associated with a VM deficit were linked to LOC/VOT (type IV loss). The data in

\footnotetext{
1 Vaina (1989) tested FfM perception on a stationary background, whereas we tested FfM perception on a dynamic background.
}

the former group show that dorsal stream areas also contribute to form-from-motion perception. We assume that the FfM perception deficits in patients with type III loss were secondary due to impaired VM perception. Alternatively, VM as well as FfM perception might have been impaired in this patient group. Linking this FfM deficit to damage in temporo-parietal cortex in close proximity to MT+/V5 agrees with neuroimaging studies that have consistently shown that MT+/V5 (that primarily codes for VM) is activated by FfM stimuli (de Jong et al., 1994; Dupont et al., 1997; Goebel et al., 1998; Tootell \& Taylor, 1995; Watson et al., 1993) and agrees with previous lesion studies showing that damage to MT+/V5 leads to impaired VM perception (Barton et al., 1995; Blanke, Landis et al., 2003; Greenlee et al., 1995; Vaina et al., 2001). Our lesion analysis suggests that damage did not extend posteriorly to include area $\mathrm{KO}$, which is an area that has been involved in FfM perception and is activated by VM and FfM stimuli (Dupont et al., 1997; Van Oostende et al., 1997; but see also Zeki, Perry, \& Bartels, 2003). Yet, based on the present lesion analysis and on the fact that the lesion overlap centered in white matter (as in most previous studies) we cannot exclude the possibility that deficits in patients with type III loss were not associated with either direct damage or disconnection-related damage to area $\mathrm{KO}$.

Our lesion analysis suggests that the severe deficits in FfM perception that were associated with VM deficits (patients with type IV loss) arise as a consequence of damage to LOC/VOT. Importantly, all patients from group IV performed the entire threshold procedure with five threshold reversals, but were incapable of discriminating even the highest threshold value that was tested with our FfM paradigm. Due to the severity of the FfM deficit and normal static form perception we hypothesize that brain damage affected neuronal populations that encode form-from-motion signals in VOT and LOC. The association with deficient VM perception suggests that LOC also codes for VM stimuli. Neuroimaging studies have consistently shown that LOC, an area that is highly active during object perception and recognition (Grill-Spector \& Malach, 2004) is also active during FfM and VM perception (Ferber et al., 2003; Gulyas et al., 1994; Wang et al., 1999). In comparison to patients with type II loss following damage to VOT, our data in patients with type IV loss suggest that additional involvement of more lateral areas in occipito-temporal areas (LOC) leads to an additional impairment of VM (that was absent in patients with damage only to VOT). Again, we cannot exclude that damage to underlying white matter in LOC in patients with type III loss led to disconnection-related damage to area MT+/V5. Interestingly our data suggest that right LOC is specialized for this association of FfM perception deficits as all patients with type IV loss suffered from right hemispheric damage.

To summarize, our data suggest that damage to right LOC may lead to a severe deficit in FfM perception that is independent from static form perception and associated with a VM perception deficit. Damage to right or left MT+/V5 may be associated with a mild to moderate deficit in FfM perception that is independent of static form perception and associated with a VM perception deficit. 


\section{Conclusion}

Our data suggest that the task of extracting FfM is mediated by ventral stream areas VOT and LOC as well as dorsal stream areas MT+/V5. Importantly, damage to these areas leads to distinct functional motion perception deficits suggesting differences in underlying functional properties of the damaged neuronal populations with respect to FfM. These data suggest a degree of specialization for FfM perception in extrastriate visual cortex that is highest in VOT, followed by LOC, and lowest at/near MT+V5. Finally, the right hemisphere plays a predominant role in the perception of FfM and VM perception that is especially important in LOC.

\section{References}

Bartels, A., \& Zeki, S. (2000). The architecture of the colour centre in the human visual brain: New results and a review. European Journal of Neuroscience, 12, 172-193.

Barton, J. S., Sharpe, J. A., \& Raymond, J. E. (1995). Retinotopic and directional defects in motion discrimination in humans with cerebral lesions. Annals of Neurology, 37, 665-675.

Blanke, O., Landis, T., Mermoud, C., Spinelli, L., \& Safran, A. B. (2003). Direction-selective motion blindness after unilateral posterior brain damage. European Journal of Neuroscience, 18, 709-722.

Blanke, O., Landis, T., Safran, A. B., \& Seeck, M. (2002). Direction-specific motion blindness induced by focal stimulation of human extrastriate cortex. European Journal of Neuroscience, 15(12), 2043-2048.

Blanke, O., Vaclavik, V., Landis, T., \& Safran, A. B. (2003). Dogphobia in a motion blind patient. Cognitive Neuropsychiatry, 8, 211-221.

Cowey, A., \& Vaina, L. M. (2000). Blindness to form from motion despite intact static form perception and motion detection. Neuropsychologia, 38(5), 566-578.

de Jong, B. M., Shipp, S., Skidmore, B., Frackowiak, R. S., \& Zeki, S. (1994). The cerebral activity related to the visual perception of forward motion in depth. Brain, 117, 1039-1054.

Dumoulin, S. O., Bittar, R. G., Kabani, N. J., Baker, C. L., Le Goualher, G., Bruce Pike, G., et al. (2000). A new anatomical landmark for reliable identification of human area V5/MT: A quantitative analysis of sulcal patterning. Cerebral Cortex, 10, 454-463.

Dupont, P., De Bruyn, B., Vandenberghe, R., Rosier, A. M., Michiels, J., Marchal, G., et al. (1997). The kinetic occipital region in human visual cortex. Cerebral Cortex, 7, 283-292.

Dupont, P., Orban, G. A., De Bruyn, B., Verbruggen, A., \& Mortelmans, L. (1994). Many areas in the human brain respond to visual motion. Journal of Neurophysiology, 72, 1420-1424.

Ferber, S., Humphrey, G. K., \& Vilis, T. (2003). The lateral occipital complex subserves the perceptual persistence of motion-defined groupings. Cerebral Cortex, 2003(13), 716-721.

Goebel, R., Khorram-Sefat, D., Muckli, L., Hacker, H., \& Singer, W. (1998). The constructive nature of vision: Direct evidence from functional magnetic resonance imaging studies of apparent motion and motion imagery. European Journal of Neuroscience, 10, 1563-1573.

Greenlee, M. W., Lang, H. J., Mergner, T., \& Seeger, W. (1995). Visual short term memory of stimulus velocity in patients with unilateral posterior brain damage. Journal of Neuroscience, 15, 2287-2300.

Grill-Spector, K., \& Malach, R. (2004). The human visual cortex. Annual Review of Neuroscience, 27, 649-677.

Gulyas, B., Heywood, C. A., Popplewell, D. A., Roland, P. E., \& Cowey, A. (1994). Visual form discrimination from color or motion cues: Func- tional anatomy by positron emission tomography. Proceedings of the National Academy of Sciences of the United States of America, 91, 99659996.

Johansson, G. (1973). Visual perception of biological motion and a model for its analysis. Perception \& Psychophysics, 14, 201-211.

Jules, B. (1971). Foundations of cyclopean perception. Chicago, IL: University of Chicago Press.

Losey, F., Safran, A. B., Mermoud, C., Michel, C., \& Landis, T. (1998). Perception visuelle du mouvement. Etude normative. Klinische Monatsblätter für Augenheilkunde, 212, 379-381.

McLeod, P., Dittrich, W., Driver, J., Perrett, D., \& Zihl, J. (1996). Preserved and impaired detection of structure from motion by a "motion-blind" patient. Visual Cognition, 3, 363-391.

Regan, D., Giaschi, D., Sharpe, J. A., \& Hong, X. H. (1992). Visual processing of motion-defined form: Selective failure in patients with parietotemporal lesions. Journal of Neuroscience, 12, 2198-2210.

Schenk, T., \& Zihl, J. (1997a). Visual motion perception after brain damage: II. Deficits in form-from-motion perception. Neuropsychologia, 35, $1299-1310$

Schenk, T., \& Zihl, J. (1997b). Visual motion perception after brain damage: I. Deficits in global motion perception. Neuropsychologia, 35, 1289-1297.

Sekuler, A. B. (1990). Motion segregation from speed differences: Evidence for nonlinear processing. Vision Research, 30, 795-1795.

Talairach, J., \& Tournoux, P. (1988). Co-planar stereotaxic atlas of the human brain. New York: Thieme.

Tootell, R. B. H., \& Taylor, J. B. (1995). Anatomical evidence for MT and additional cortical visual areas in humans. Cerebral Cortex, 1, 3955.

Van Oostende, S., Sunaert, S., Van Hecke, P., Marchal, G., \& Orban, G. A. (1997). The kinetic occipital (KO) region in man: An fMRI study. Cerebral Cortex, 7, 690-701.

Vaina, L. M. (1989). Selective impairment of visual motion interpretation following lesions of the right occipito-parietal area in humans. Biological Cybernetics, 61, 347-359.

Vaina, L. M., Cowey, A., Eskew, R. T., LeMay, M., \& Kemper, T. (2001). Regional cerebral correlates of global motion perception: Evidence from unilateral cerebral brain damage. Brain, 124, 310-321.

Vaina, L. M., \& Gross, C. G. (2004). Perceptual deficits in patients with impaired recognition of biological motion after temporal lobe lesions. Proceedings of the National Academy of Science, 101(48), 16947-16951.

Vaina, L. M., Lemay, M., Bienfang, D. C., Choi, A. Y., \& Nakayama, K. (1990). Intact "biological motion" and "structure from motion" perception in a patient with impaired motion mechanisms: A case study. Visual Neuroscience, 5(4), 353-369.

Vaina, L. M., Cowey, A., Jakab, M., \& Kikinis, R. (2005). Deficits of motion integration and segregation in patients with unilateral extrastriate lesions. Brain, 128, 2134-2145.

Wang, J., Zhou, T., Qiu, M., Du, A., Cai, K., Wang, Z., et al. (1999). Relationship between ventral stream for object vision and dorsal stream for spatial vision: An fMRI+ERP study. Human Brain Mapping, 8, 170181.

Watson, J. D. G., Myers, R., Frackowiak, R. S., Hajnal, J. V., Woods, R. P., Mazziotta, J. C., et al. (1993). Area V5 of the human brain: Evidence from a combined study using positron emission tomography and magnetic resonance imaging. Cerebral Cortex, 3, 79-94.

Wist, E. R., Ehrenstein, W. H., \& Schrauf, M. (1998). A computer-assisted test for the electrophysiological and psychophysical measurement of dynamic visual function based on motion contrast. Journal of Neuroscience Methods, 80, 41-47.

Zeki, S., Perry, R. J., \& Bartels, A. (2003). The processing of kinetic contours in the brain. Cerebral Cortex, 13(2), 189-202.

Zihl, J., von Cramon, D., \& Mai, N. (1983). Selective disturbance of movement vision after bilateral brain damage. Brain, 106, 313-340. 